\title{
Comparison of Combinatorial and Continuous Frameworks For The Beam Angle Optimization Problem in IMRT
}

\author{
Humberto Rocha ${ }^{1,2} 0000-0002-5981-4469$, Joana Dias ${ }^{1,2} 0000-0003-2517-7905$, \\ Tiago Ventura ${ }^{3} 0000-0003-1562-0387$, \\ Brígida Ferreira ${ }^{4,5} 0000-0001-7988-7545$, and Maria do Carmo \\ Lopes $^{3,5} 0000-0001-7339-7342$ \\ 1 CeBER and Faculdade de Economia, Universidade de Coimbra, \\ 3004-512 Coimbra, Portugal \\ 2 INESC-Coimbra, \\ 3030-290 Coimbra, Portugal \\ 3 Serviço de Física Médica, IPOC-FG, EPE, \\ 3000-075 Coimbra, Portugal \\ 4 ESS.PP, Politécnico do Porto \\ 4400-330 Vila Nova de Gaia, Portugal \\ 5 I3N.UA, Universidade de Aveiro \\ 3810-193 Aveiro, Portugal \\ hrocha@mat.uc.pt, joana@fe.uc.pt, tiagoventura@ipocoimbra.min-saude.pt, \\ bcf@ess.ipp.pt, mclopes@ipocoimbra.min-saude.pt
}

\begin{abstract}
Radiation therapy (RT) is used nowadays for the majority of cancer patients. A technologically advanced type of RT is IMRT intensity-modulated radiation therapy. With this RT modality the cancerous cells of the patient can be irradiated using non-uniform radiation maps delivered from different beam directions. Although non-uniform radiation maps allow, by themselves, an enhanced sparing of the neighboring healthy organs while properly irradiating the tumor with the prescribed dose, selection of appropriate irradiation directions play a decisive role on these conflicting tasks: deliver dose to the tumor while preventing (too much) dose to be deposited in the surrounding tissues. This paper focus on the problem of choosing the best set of irradiation directions, known as beam angle optimization (BAO) problem. Two completely different mathematical formulations of this problem can be found in the literature. A combinatorial formulation, widely used and addressed by many different algorithms and strategies, and a continuous formulation proposed by the authors and addressed by derivative-free algorithms. In this paper, a comparison of two of the most successful strategies to address each one of these formulations is done resorting to a set of ten clinical nasopharyngeal tumor cases already treated at the Portuguese Institute of Oncology of Coimbra.
\end{abstract}

Keywords: IMRT, Beam Angle Optimization, Combinatorial Optimization, Derivative-free Optimization 


\section{Introduction}

Cancer incidence and prevalence is continuously growing. The majority of the cancer patients is nowadays treated with radiation therapy (RT), either to kill the cancer cells or to palliate the symptoms. A technologically advanced type of $\mathrm{RT}$ is intensity-modulated radiation therapy (IMRT). A multileaf collimator is used in IMRT to transform the radiation beam into a number of discrete small sub-beams called beamlets. The optimal intensities of these beamlets are calculated independently, in a large-scale optimization problem called fluence map optimization (FMO) problem, leading to non-uniform radiation maps. Typically, a predefined number of non-uniform radiation maps intersects the tumor from different irradiation directions. Although non-uniform radiation maps allow, by themselves, an enhanced sparing of the neighboring healthy organs while properly irradiating the tumor with the prescribed dose, selection of appropriate irradiation directions play a decisive role on these conflicting tasks: deliver dose to the tumor while preventing (too much) dose to be deposited in the surrounding tissues. The optimal selection of beam irradiation directions, known as beam angle optimization (BAO) problem, is a very difficult optimization problem.

Two completely different mathematical formulations of the BAO problem can be found in the literature. A combinatorial formulation, widely used, considers a discrete sample of all continuous beam angle directions. This formulation leads to a NP-hard optimization problem [3]. A large number of different algorithms have been used to speed up the searches, including gradient search [8], neighborhood search [1], simulated annealing [10], response surface approaches [2], branch-and-prune [12] or hybrid approaches [5]. Iterative BAO is a successful combinatorial strategy used in practice that adds one beam at a time, sequentially, to a treatment plan, reducing the total number of possible combinations significantly [3]. Alternatively, a continuous formulation has been proposed by the authors, considering all continuous beam angle directions. This formulation leads to a highly non-convex optimization problem with many local minima on a continuous search space. Figure 1 illustrates the non-convex nature of a twodimension BAO problem for a nasopharyngeal tumor case. Due to the curse of dimensionality, the number of local minima will increase exponentially for larger dimensions. The continuous search space of the BAO problem has been explored using derivative-free optimization frameworks $[14,15,16,17]$.

This paper compares two of the most successful strategies used to address the continuous and the combinatorial BAO formulations: a parallel multistart derivative-free framework that explores thoroughly the BAO problem continuous search space is compared to an iterative BAO framework that would obtain a theoretical upper limit of the treatment plan quality [19]. A set of ten clinical cases of nasopharyngeal (intra-cranial) tumors treated at the Portuguese Institute of Oncology of Coimbra (IPOC) is used to test and discuss the benefits of our continuous approach against the benchmark combinatorial approach. The paper is organized as follows. In the next section we present a continuous and a combinatorial formulation of the BAO problem. In section three we describe a parallel multistart derivative-free framework for the BAO problem. 


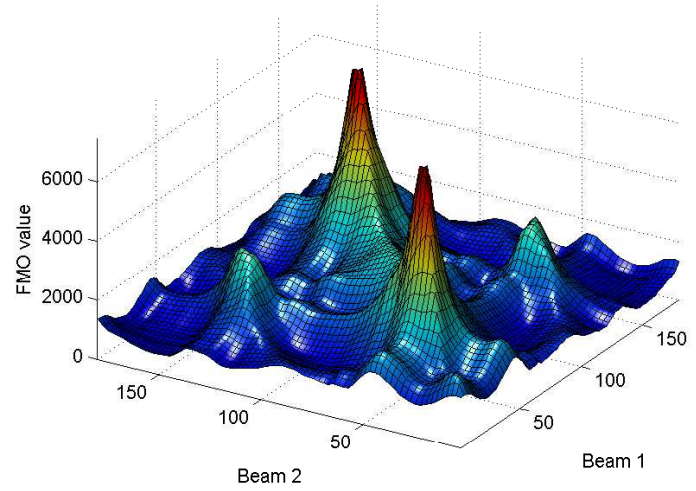

Fig. 1. BAO surface for a nasopharyngeal tumor case considering only two coplanar beam irradiation directions.

Computational tests are presented in section four. In the last section we have the conclusions.

\section{Mathematical Formulation of the BAO Problem}

The aim of BAO is to determine the best ensemble of beam angle directions to irradiate the patient. Here, coplanar beams are considered, i.e. beam directions lay on the plane of rotation of the linear accelerator around the patient that lays in a fix positioned couch. It is assumed that the treatment planner defines, a priori, the number of beam irradiation directions to be $n$. In order to assess the quality of an $n$-beam angle ensemble, $\theta_{1}, \ldots, \theta_{n}$, the optimal value of the FMO problem obtained for that beam angle ensemble, $f\left(\theta_{1}, \ldots, \theta_{n}\right)$, is used. A mathematical formulation for the BAO problem can then consider that the best ensemble of beam directions is obtained for the (FMO) function's minimum:

$\min f\left(\theta_{1}, \ldots, \theta_{n}\right)$

s.t. $\theta_{1}, \ldots, \theta_{n} \in \Theta$, where $\Theta$ is the set of all possible beam angles.

For a combinatorial BAO formulation, the interval of possible gantry angles, $\left[0^{\circ}, 360^{\circ}\right.$ [, is discretized into evenly spaced angles. For example, for an angle increment of one degree, the set of all candidate beam angles $\Theta$ of Eq. (1) corresponds to the set of 360 beam angles $\{0,1, \ldots, 359\}$. For a continuous BAO formulation, the interval of possible gantry angles $\left[0^{\circ}, 360^{\circ}\right.$ [ is considered. Note that the beam angle directions $-10^{\circ}$ and $350^{\circ}$ are the same as well as beam angle 
directions $370^{\circ}$ and $10^{\circ}$. Thus, we can consider $\Theta=\mathbb{R}^{n}$ and avoid a bounded formulation.

Regardless of using a combinatorial or a continuous BAO formulation, the quality of each beam ensemble is assessed through the FMO optimal value. The resolution of the FMO problem requires the accurate assessment of the radiation dose distribution, measured in Gray (Gy), for each irradiated structure of the patient. The volume of each structure is discretized into small volume elements called voxels. The dose is calculated for each individual voxel using the principle of superposition, i.e., adding the dose from all beamlets that reach each individual voxel. Using the superposition principle, i.e., considering the contribution of each beamlet, the dose is computed for each voxel. Typically, for a nasopharyngeal treatment plan the number of beamlets $\left(N_{b}\right)$ reaches the hundreds while the number of voxels $\left(N_{v}\right)$ reaches the tens of thousands. For optimization purposes, a dose matrix $D$ is constructed considering the beamlet intensities and by indexing each column to a given beamlet and each row to a given voxel. Thus, the dose deposited in voxel $i$ by beamlet $j$ is stored in row $i$ and column $j$ of matrix $D$. The total dose received by voxel $i$ is the sum of the doses of all beamlets that reach voxel $i$, i.e., $\sum_{j=1}^{N_{b}} D_{i j} w_{j}$, where $w_{j}$ is the intensity (or weight) of beamlet $j$. The main difficulty in solving the FMO problem is the dimension of the dose matrix that originates large-scale optimization problems.

Many different mathematical models and optimization procedures have been presented for the FMO problem, including linear models [18], nonlinear models [7], a priori multicriteria models [6], a posteriori multicriteria models [13], particle swarm optimization models [20] and fuzzy inference systems models [11]. Here, we use the following convex penalty function voxel-based nonlinear model [1]:

$$
\begin{aligned}
& \min _{w} \sum_{i=1}^{N_{v}}\left[\underline{\lambda}_{i}\left(T_{i}-\sum_{j=1}^{N_{b}} D_{i j} w_{j}\right)_{+}^{2}+\bar{\lambda}_{i}\left(\sum_{j=1}^{N_{b}} D_{i j} w_{j}-T_{i}\right)_{+}^{2}\right] \\
& \text { s.t. } \quad w_{j} \geq 0, j=1, \ldots, N_{b},
\end{aligned}
$$

where $\bar{\lambda}_{i}$ and $\underline{\lambda}_{i}$ are weights that penalize overdose and underdose of voxel $i, T_{i}$ is the tolerance/prescribed dose for voxel $i$ and $(\cdot)_{+}=\max \{0, \cdot\}$. This model penalizes the square difference between the tolerance/prescribed dose and the received dose by each voxel, implying that small differences from the tolerance/prescribed dose (overdose or underdose) may be clinically tolerated while larger differences from the tolerance/prescribed dose are decreasingly accepted.

The discussion of the most appropriate FMO problem formulation/resolution to be embedded in a BAO framework is out of the scope of this study. Furthermore, the FMO model is used as a black-box function. Thus, the conclusions drawn regarding continuous or combinatorial BAO formulations/resolutions are valid regardless of the FMO formulation/resolution considered. 


\section{Parallel Multistart Derivative-free Optimization Framework}

The multistart strategy designed to address the continuous BAO formulation takes advantage of the peculiarities of this particular space. As the order of the irradiation directions of a beam ensemble is not important, the continuous BAO search space has symmetry features, which allows a large reduction of the space to be explored by simply keeping the beam directions sorted for each beam ensemble [17]. In order to sample this reduced search space in an appropriate manner, all possible combinations of sorted beam ensembles divided by the 4 quadrants will be considered as starting beam ensembles (iterates or points). E.g., for a continuous three-dimensional BAO search space, all possible threebeam directions distribution by the four quadrants are illustrated in Fig. 2(a). Each of these three-beam ensemble corresponds to a starting point placed in the different painted cubes illustrated in Fig. 2(b). For continuous tree-dimension BAO search space only $\frac{1}{4}$ of the entire search space $[0,360]^{3}$ is explored. For $n$-beam angle ensembles, the total number of (hyper)cubes of the entire search space is $4^{n}$ while the number of (hyper)cubes of the reduced search space, which corresponds to the number of possible distributions of $n$ sorted beam angles by the 4 quadrants is the combination with repetition of $\left(\begin{array}{c}n+4-1 \\ 4\end{array}\right)=\frac{(n+4-1) !}{4 !(n-1) !}$. For continuous $n$-dimension BAO search space only $\frac{1}{2^{n}}$ of the entire search space $[0,360]^{n}$ is explored.

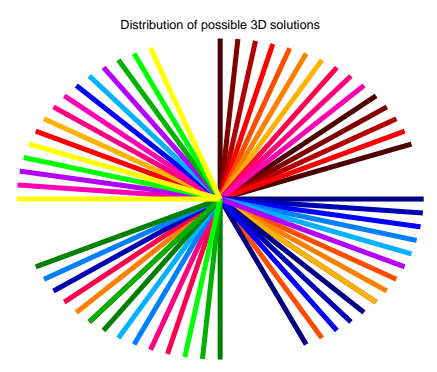

(a)

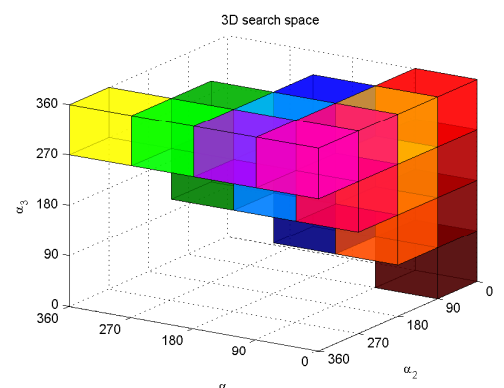

(b)

Fig. 2. Three beam directions distribution by the four quadrants - 2(a) and the corresponding cubes in the search space $[0,360]^{3}-2(b)$.

After setting the starting points, $\mathbf{x}_{i}^{0} \in[0,360]^{n}, i=1, \ldots, N$, one for each hypercube of the reduced search space, the objective function value is evaluated at each of these initial beam ensembles. The best solutions and corresponding objective function values found so far for each region (hypercube) are assigned to these initial points and corresponding function values: $\mathbf{x}_{i}^{*}=\mathbf{x}_{i}^{0}, i=1, \ldots, N$; $f_{i}^{*}=f\left(\mathbf{x}_{i}^{0}\right), i=1, \ldots, N$. A local procedure is then used to locally improve 
that value. Two important aspects of a parallel multistart procedure in a multimodal search space must be cautioned. First, as different search procedures coexist in time, the same region may end up being explored by different local search procedures wasting precious computational time. In order to avoid that, each hypercube can only be explored by a single local search procedure at a time. When the outcome of different local search procedures lay in the same hypercube, only the local search yielding the iterate with lowest function value remain active. Information of the regions that have active local searches is stored using a boolean vector, Active $\mathbf{A}_{N \times 1}$, that is updated at the end of each iteration. Second, due to the highly non-convex nature of the search space, derivativefree local search algorithms are advisable. Pattern search methods (PSM) were previously selected for the resolution of the continuous BAO problem as they have the ability to avoid local entrapment and need a reduced number of function (FMO) evaluations to converge $[14,15,16]$. Pattern search methods as described in Rocha et al. $[14,15,16]$ are used as local search procedure. Algorithm 1 displays the parallel multistart PSM algorithm.

\section{Computational Results}

A set of ten clinical examples of nasopharyngeal tumor cases already treated at IPOC were used to test the different approaches. For the nasopharyngeal tumor cases in study, two different planning target volumes (PTVs) were considered, $P T V_{70}$ and $P T V_{59.4}$, corresponding to two different levels of prescribed radiation doses. The organs at risk (OARs) considered were the brainstem, the spinal cord, the oral cavity and the parotid glands. OAR tolerance doses and prescribed doses for the PTVs are depicted at Table 1. The tolerance dose considered for the brainstem and the spinal cord is the maximum dose as these are serial type organs, i.e., organs whose function is jeopardized even if only a small portion is injured. The tolerance dose for the parotid glands, the larger salivary gland, and the oral cavity, that contains the remaining salivary glands, is the mean dose because the salivary glands are parallel type organs, i.e., organs whose function is not jeopardized if only a small portion is injured.

Our tests were performed on a 2.2 Ghz Intel Xeon 8-core computer workstation with 25 GB RAM. The nonlinear convex FMO formulation (Eq. (1)) was addressed using a trust-region-reflective algorithm (fmincon) from Optimization Toolbox of MATLAB (R2016a). CERR [9] was used to import the patients' computed tomography $(\mathrm{CT})$ sets with the considered structures delineated. This freeware research software allows the computation of the necessary dosimetric data for treatment planning optimization as well as convenient visualization and analysis of the treatment plans obtained. QIB, the pencil beam algorithm of CERR, was used for dose calculations. An automated dose calculation proce-

dure for each beam ensemble was developed instead of using the menu bar of CERR.

Typically, nasopharyngeal tumors are treated with five to nine equispaced coplanar beam ensembles. Since the importance of appropriate selection of beam 


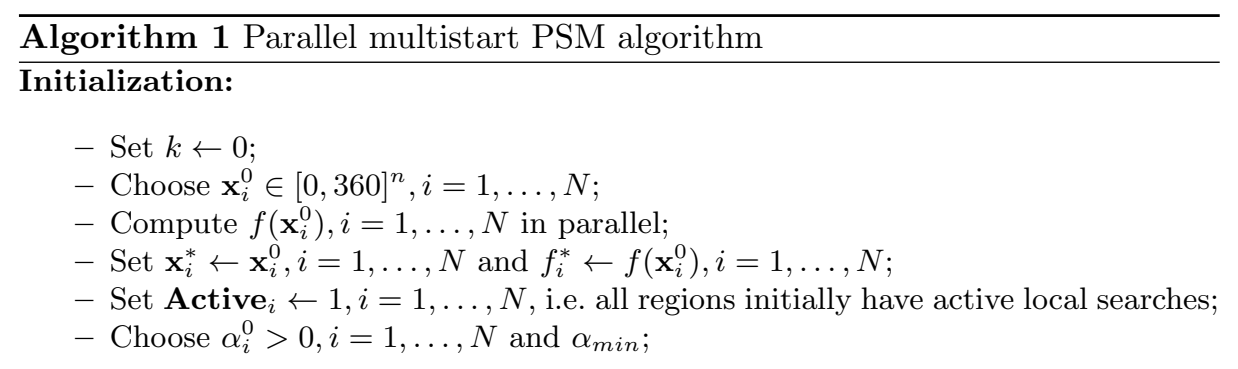

\section{Iteration:}

1. Use PSM to locally explore the hypercubes with active local search;

2. For hypercubes $i$ with active local search do

$$
\begin{aligned}
& \text { If } f\left(\mathbf{x}_{i}^{k}\right)<f\left(\mathbf{x}_{i}^{*}\right) \text { then } \\
& \text { If } \mathbf{x}_{i}^{k} \text { is in hypercube } i \text { then } \\
& \mathbf{x}_{i}^{*} \leftarrow \mathbf{x}_{i}^{k} ; \\
& f_{i}^{*} \leftarrow f\left(\mathbf{x}_{i}^{k}\right) ; \\
& \text { Else }
\end{aligned}
$$

Active $_{i} \leftarrow 0$

Determine hypercube $j \neq i$ where $\mathbf{x}_{i}^{k}$ is;

If $f\left(\mathbf{x}_{i}^{k}\right)<f\left(\mathbf{x}_{j}^{*}\right)$ then

$\mathbf{x}_{j}^{*} \leftarrow \mathbf{x}_{i}^{k}$

$f_{j}^{*} \leftarrow f\left(\mathbf{x}_{i}^{k}\right)$

Active $_{j} \leftarrow 1$

Else

$$
\begin{aligned}
& \alpha_{i}^{k+1} \leftarrow \frac{\alpha_{i}^{k}}{2} \\
& \text { If } \alpha_{i}^{k+1}<\alpha_{\min } \text { then }
\end{aligned}
$$

Active $_{i} \leftarrow 0$;

3. If there exists active hypercubes go to first step and set $k \leftarrow k+1$. 
Table 1. Prescribed doses and tolerance doses for tumor volumes and OARs.

\begin{tabular}{lccc}
\hline Structure & Mean dose & Max dose Prescribed dose \\
\hline PTV $_{\mathbf{7 0}}$ & - & - & $70.0 \mathrm{~Gy}$ \\
PTV $_{\mathbf{5 9 . 4}}$ & - & - & $59.4 \mathrm{~Gy}$ \\
Brainstem & - & $54 \mathrm{~Gy}$ & - \\
Spinal cord & - & $45 \mathrm{~Gy}$ & - \\
Oral cavity & $30 \mathrm{~Gy}$ & - & - \\
Left parotid & $26 \mathrm{~Gy}$ & - & - \\
Right parotid & $26 \mathrm{~Gy}$ & - & - \\
\hline
\end{tabular}

directions increases for lower number of beam angles, we consider treatment plans with five coplanar beams. Thus, using the multistart PSM framework, five-beam treatment plans were obtained and denoted MultistartBAO. The initial step-size considered was $\alpha^{0}=2^{5}=32$ and the minimal value allowed was one, defining the stopping criteria. By choosing a power of two for initial step-size, as step-size remains the same at successful iterations and is halved at unsuccessful ones, the beam directions remain integer until the stopping criteria when the step-size becomes a rational number. Despite only integer irradiation directions are tested, it is worth to highlight that the continuous BAO space is explored which is fundamentally different from a combinatorial approach.

Iterative BAO is a successful strategy used in practice [6]. Furthermore, this strategy would obtain a theoretical upper limit of the treatment plan quality [19]. Treatment plans obtained by Wild et al. [19], called $4 \pi$ and corresponding to a theoretical upper limit of a plan's quality, were obtained using iterative BAO considering noncoplanar beam orientations for a 5 degree angular spacing. In this study, Multistart $B A O$ plans were compared with five-beam treatment plans obtained using iterative $\mathrm{BAO}$ and denoted IterativeBAO. A discrete set of 360 beam directions, $\{0,1,2, \ldots, 359\}$, was used by considering a one degree angular spacing. In iterative BAO, beams are added sequentially one at a time to a treatment plan. The first beam is determined by computing the optimal FMO value of all one-beam ensembles for each possible beam direction. The one-beam ensemble leading to the lowest optimal FMO value is selected. Given a beam ensemble with $n-1$ beam irradiation directions, the next beam direction, $n$ th, is determined by computing the optimal FMO value of all $n$-beam ensembles obtained by adding each of the remaining beam directions to the the $n-1$-beam ensemble. The $n$-beam ensemble selected corresponds to the one yielding the lowest FMO value. Thus, obtaining a five-beam ensemble requires the computation of $360+359+358+357+356=1790$ FMO optimal values. Nevertheless, this greedy strategy reduces the number of FMO problem resolutions compared to other combinatorial BAO approaches.

Treatment plans obtained using optimized beam ensembles were also compared with five-beam coplanar equispaced treatment plans, denoted Equi. The objective of these comparisons is to benchmark the treatment plans with optimal 
Table 2. Results of the beam angle optimization processes.

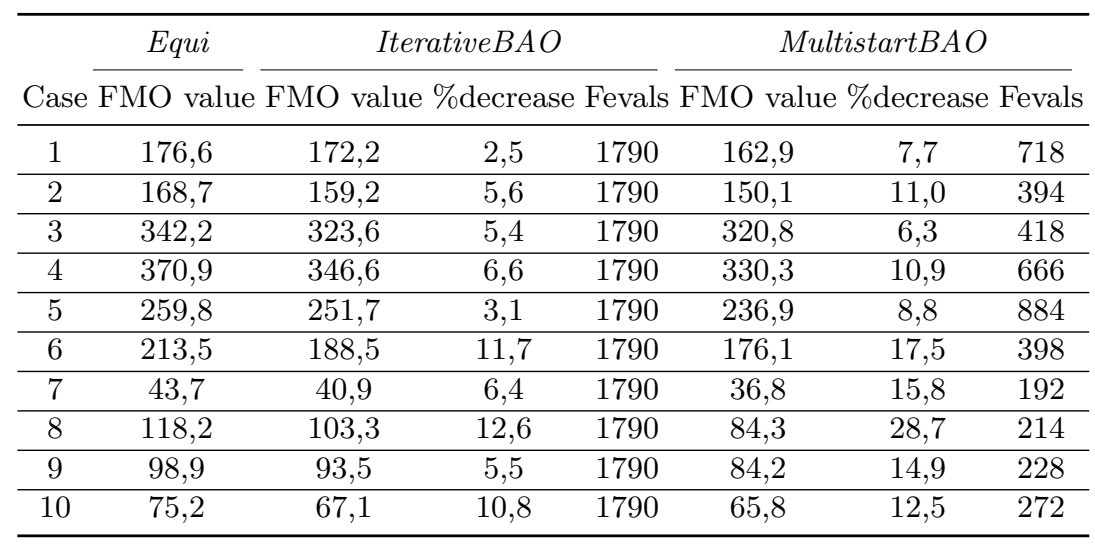

beam angle ensembles with treatment plans commonly used in clinical practice. Table 2 displays the results of the BAO processes in terms of final FMO value, the measure considered for quality assessment of a beam ensemble. Compared to the FMO value of the Equi treatment plans, MultistartBAO obtained an average reduction of the FMO value of $13,4 \%$, clearly outperforming Iterative $B A O$ that obtained an average redution of 7,0\%. Furthermore, MultistartBAO required an average of 438 function evaluations which is about four times less than the number required by IterativeBAO. Average computational time required by $\mathrm{Mul}$ tistart $B A O$ was three hours while Iterative $B A O$ spent an average of nine hours (also computed in parallel).

Despite the good results in terms of optimal FMO value improvement, treatment plan's quality can be acknowledged by different dose metrics. One of the most important target metrics is the tumor coverage, i.e. the percent of the tumor volume that receives at least $95 \%$ of the prescribed dose. Existence of coldspots, i.e. percentage of the tumor volume receiving less than $93 \%$ of the prescribed dose, and occurrence of hotspots, i.e. percentage of the tumor volume receiving more than $110 \%$ of the prescribed dose, are also metrics of interest. These three target metrics, displayed in Table 3, show that MultistartBAO outperforms both Iterative $B A O$ and Equi treatment plans concerning tumor coverage metrics.

Metrics usually screened for OARs are mean and/or maximum doses, depending if the organ has a parallel or serial architecture, respectively. Table 4 depicts organ sparing results. For the brainstem and the spinal cord, serial organs, the maximum dose is displayed. It can be verified that treatment plans with optimized beam directions always comply with the prescribed maximum doses while Equi treatment plans fail to do so in some cases. For oral cavity and parotids, parallel organs, the mean dose is displayed. Improved sparing of oral cavity and parotids is clearly obtained by treatment plans with optimal five-beam ensembles. Compared to the Equi treatment plans, in average, Multi- 
Table 3. Target coverage obtained by treatment plans.

\begin{tabular}{|c|c|c|c|c|}
\hline Case & Target coverage & Multistart $B A O$ & Iterative $B A O$ & Equi \\
\hline \multirow{6}{*}{1} & $\mathrm{PTV}_{70}$ at $95 \%$ volume & $66.5 \mathrm{~Gy}$ & $66.5 \mathrm{~Gy}$ & $66.1 \mathrm{~Gy}$ \\
\hline & $\mathrm{PTV}_{70} \%>93 \%$ of $\mathrm{Rx}(\%)$ & 99.1 & 98.9 & 98.0 \\
\hline & $\mathrm{PTV}_{70} \%>110 \%$ of $\mathrm{Rx}(\%)$ & 0.0 & 0.0 & 0.0 \\
\hline & $\mathrm{PTV}_{59.4}$ at $95 \%$ volume & 57.5 Gy & 57.1 Gy & $57.3 \mathrm{~Gy}$ \\
\hline & $\mathrm{PTV}_{59.4} \%>93 \%$ of $\mathrm{Rx}(\%)$ & 97.4 & 96.7 & 97.0 \\
\hline & $\mathrm{PTV}_{59.4} \%>110 \%$ of $\mathrm{Rx}(\%)$ & 15.1 & 15.4 & 14.8 \\
\hline \multirow{6}{*}{2} & $\mathrm{PTV}_{70}$ at $95 \%$ volume & $67.5 \mathrm{~Gy}$ & $67.1 \mathrm{~Gy}$ & $67.3 \mathrm{~Gy}$ \\
\hline & $\mathrm{PTV}_{70} \%>93 \%$ of $\mathrm{Rx}(\%)$ & 99.9 & 98.9 & 99.5 \\
\hline & $\mathrm{PTV}_{70} \%>110 \%$ of $\mathrm{Rx}(\%)$ & 0.0 & 0.0 & 0.0 \\
\hline & $\mathrm{PTV}_{59.4}$ at $95 \%$ volume & $54.7 \mathrm{~Gy}$ & $54.7 \mathrm{~Gy}$ & $53.5 \mathrm{~Gy}$ \\
\hline & $\mathrm{PTV}_{59.4} \%>93 \%$ of $\mathrm{Rx}(\%)$ & 94.4 & 94.4 & 93.0 \\
\hline & $\mathrm{PTV}_{59.4} \%>110 \%$ of $\mathrm{Rx}(\%)$ & 5.3 & 5.3 & 5.6 \\
\hline \multirow{6}{*}{3} & $\mathrm{PTV}_{70}$ at $95 \%$ volume & $65.7 \mathrm{~Gy}$ & $65.5 \mathrm{~Gy}$ & $65.1 \mathrm{~Gy}$ \\
\hline & $\mathrm{PTV}_{70} \%>93 \%$ of $\mathrm{Rx}(\%)$ & 96.6 & 95.9 & 94.9 \\
\hline & $\mathrm{PTV}_{70} \%>110 \%$ of $\mathrm{Rx}(\%)$ & 0.0 & 0.0 & 0.0 \\
\hline & $\mathrm{PTV}_{59.4}$ at $95 \%$ volume & $54.1 \mathrm{~Gy}$ & $54.1 \mathrm{~Gy}$ & $53.3 \mathrm{~Gy}$ \\
\hline & $\mathrm{PTV}_{59.4} \%>93 \%$ of $\mathrm{Rx}(\%)$ & 93.9 & 93.7 & 93.3 \\
\hline & $\mathrm{PTV}_{59.4} \%>110 \%$ of $\mathrm{Rx}(\%)$ & 25.4 & 25.4 & 25.0 \\
\hline \multirow{6}{*}{4} & $\mathrm{PTV}_{70}$ at $95 \%$ volume & $68.3 \mathrm{~Gy}$ & $68.3 \mathrm{~Gy}$ & $68.3 \mathrm{~Gy}$ \\
\hline & $\mathrm{PTV}_{70} \%>93 \%$ of $\mathrm{Rx}(\%)$ & 99.5 & 99.7 & 99.7 \\
\hline & $\mathrm{PTV}_{70} \%>110 \%$ of $\mathrm{Rx}(\%)$ & 0.0 & 0.0 & 0.0 \\
\hline & $\mathrm{PTV}_{59.4}$ at $95 \%$ volume & $53.5 \mathrm{~Gy}$ & $53.5 \mathrm{~Gy}$ & $51.3 \mathrm{~Gy}$ \\
\hline & $\mathrm{PTV}_{59.4} \%>93 \%$ of $\mathrm{Rx}(\%)$ & 93.2 & 93.1 & 91.4 \\
\hline & $\mathrm{PTV}_{59.4} \%>110 \%$ of $\mathrm{Rx}(\%)$ & 18.5 & 18.7 & 19.5 \\
\hline \multirow{6}{*}{5} & $\mathrm{PTV}_{70}$ at $95 \%$ volume & $67.5 \mathrm{~Gy}$ & $67.3 \mathrm{~Gy}$ & $67.1 \mathrm{~Gy}$ \\
\hline & $\mathrm{PTV}_{70} \%>93 \%$ of $\mathrm{Rx}(\%)$ & 99.6 & 99.4 & 99.1 \\
\hline & $\mathrm{PTV}_{70} \%>110 \%$ of $\mathrm{Rx}(\%)$ & 0.0 & 0.0 & 0.0 \\
\hline & $\mathrm{PTV}_{59.4}$ at $95 \%$ volume & $52.7 \mathrm{~Gy}$ & $51.9 \mathrm{~Gy}$ & $51.5 \mathrm{~Gy}$ \\
\hline & $\mathrm{PTV}_{59.4} \%>93 \%$ of $\mathrm{Rx}(\%)$ & 93.1 & 92.4 & 92.1 \\
\hline & $\mathrm{PTV}_{59.4} \%>110 \%$ of $\mathrm{Rx}(\%)$ & 7.7 & 7.8 & 8.0 \\
\hline \multirow{6}{*}{6} & $\mathrm{PTV}_{70}$ at $95 \%$ volume & 64.9 Gy & $64.7 \mathrm{~Gy}$ & $64.9 \mathrm{~Gy}$ \\
\hline & $\mathrm{PTV}_{70} \%>93 \%$ of $\mathrm{Rx}(\%)$ & 99.4 & 94.2 & 94.3 \\
\hline & $\mathrm{PTV}_{70} \%>110 \%$ of $\mathrm{Rx}(\%)$ & 0.0 & 0.0 & 0.0 \\
\hline & $\mathrm{PTV}_{59.4}$ at $95 \%$ volume & $58.3 \mathrm{~Gy}$ & $58.3 \mathrm{~Gy}$ & $57.9 \mathrm{~Gy}$ \\
\hline & $\mathrm{PTV}_{59.4} \%>93 \%$ of $\mathrm{Rx}(\%)$ & 98.2 & 98.2 & 97.7 \\
\hline & $\mathrm{PTV}_{59.4} \%>110 \%$ of $\mathrm{Rx}(\%)$ & 6.2 & 6.2 & 5.9 \\
\hline \multirow{6}{*}{7} & $\mathrm{PTV}_{70}$ at $95 \%$ volume & $67.7 \mathrm{~Gy}$ & $67.5 \mathrm{~Gy}$ & $67.7 \mathrm{~Gy}$ \\
\hline & $\mathrm{PTV}_{70} \%>93 \%$ of $\mathrm{Rx}(\%)$ & 99.3 & 99.3 & 99.2 \\
\hline & $\mathrm{PTV}_{70} \%>110 \%$ of $\mathrm{Rx}(\%)$ & 0.0 & 0.0 & 0.0 \\
\hline & $\mathrm{PTV}_{59.4}$ at $95 \%$ volume & $57.5 \mathrm{~Gy}$ & $57.5 \mathrm{~Gy}$ & $57.3 \mathrm{~Gy}$ \\
\hline & $\mathrm{PTV}_{59.4} \%>93 \%$ of $\mathrm{Rx}(\%)$ & 98.2 & 97.9 & 97.9 \\
\hline & $\mathrm{PTV}_{59.4} \%>110 \%$ of $\mathrm{Rx}(\%)$ & 0.3 & 0.3 & 0.3 \\
\hline \multirow{6}{*}{8} & $\mathrm{PTV}_{70}$ at $95 \%$ volume & $67.3 \mathrm{~Gy}$ & $67.3 \mathrm{~Gy}$ & $67.1 \mathrm{~Gy}$ \\
\hline & $\mathrm{PTV}_{70} \%>93 \%$ of $\mathrm{Rx}(\%)$ & 98.8 & 98.5 & 98.3 \\
\hline & $\mathrm{PTV}_{70} \%>110 \%$ of $\mathrm{Rx}(\%)$ & 0.0 & 0.0 & 0.0 \\
\hline & $\mathrm{PTV}_{59.4}$ at $95 \%$ volume & $57.5 \mathrm{~Gy}$ & $56.9 \mathrm{~Gy}$ & $56.9 \mathrm{~Gy}$ \\
\hline & $\mathrm{PTV}_{59.4} \%>93 \%$ of $\mathrm{Rx}(\%)$ & 98.0 & 97.0 & 97.0 \\
\hline & $\mathrm{PTV}_{59.4} \%>110 \%$ of $\mathrm{Rx}(\%)$ & 0.9 & 1.0 & 1.1 \\
\hline \multirow{6}{*}{9} & $\mathrm{PTV}_{70}$ at $95 \%$ volume & $66.9 \mathrm{~Gy}$ & $66.9 \mathrm{~Gy}$ & $66.3 \mathrm{~Gy}$ \\
\hline & $\mathrm{PTV}_{70} \%>93 \%$ of $\mathrm{Rx}(\%)$ & 98.1 & 97.8 & 96.9 \\
\hline & $\mathrm{PTV}_{70} \%>110 \%$ of $\mathrm{Rx}(\%)$ & 0.0 & 0.0 & 0.0 \\
\hline & $\mathrm{PTV}_{59.4}$ at $95 \%$ volume & 57.7 Gy & $57.5 \mathrm{~Gy}$ & $57.3 \mathrm{~Gy}$ \\
\hline & $\mathrm{PTV}_{59.4} \%>93 \%$ of $\mathrm{Rx}(\%)$ & 97.9 & 97.7 & 97.7 \\
\hline & $\mathrm{PTV}_{59.4} \%>110 \%$ of $\mathrm{Rx}(\%)$ & 0.2 & 0.3 & 0.5 \\
\hline \multirow{6}{*}{10} & $\mathrm{PTV}_{70}$ at $95 \%$ volume & $66.7 \mathrm{~Gy}$ & $66.9 \mathrm{~Gy}$ & $66.7 \mathrm{~Gy}$ \\
\hline & $\mathrm{PTV}_{70} \%>93 \%$ of $\mathrm{Rx}(\%)$ & 98.1 & 98.5 & 97.9 \\
\hline & $\mathrm{PTV}_{70} \%>110 \%$ of $\mathrm{Rx}(\%)$ & 0.0 & 0.0 & 0.0 \\
\hline & $\mathrm{PTV}_{59.4}$ at $95 \%$ volume & $57.3 \mathrm{~Gy}$ & $57.1 \mathrm{~Gy}$ & $57.1 \mathrm{~Gy}$ \\
\hline & $\mathrm{PTV}_{59.4} \%>93 \%$ of $\mathrm{Rx}(\%)$ & 97.4 & 97.4 & 97.2 \\
\hline & $\mathrm{PTV}_{59.4} \%>110 \%$ of $\mathrm{Rx}(\%)$ & 0.9 & 0.9 & 0.8 \\
\hline
\end{tabular}


Table 4. OARs sparing obtained by treatment plans.

\begin{tabular}{|c|c|c|c|c|c|c|c|}
\hline \multirow[b]{2}{*}{ Case } & \multirow[b]{2}{*}{ OAR } & \multicolumn{3}{|c|}{ Mean Dose (Gy) } & \multicolumn{3}{|c|}{ Max Dose (Gy) } \\
\hline & & Multistart $B A O$ & Iterative $B A O$ & Equi & Multistart $B A O$ & Iterative $B A O$ & Equi \\
\hline \multirow{5}{*}{1} & Spinal cord & - & - & - & 38.7 & 42.1 & 39.1 \\
\hline & Brainstem & - & - & - & 52.6 & 52.4 & 53.8 \\
\hline & Right parotid & 22.3 & 23.7 & 24.4 & - & - & - \\
\hline & Left parotid & 24.2 & 24.0 & 25.4 & - & - & - \\
\hline & Oral Cavity & 25.6 & 27.1 & 28.6 & - & - & - \\
\hline \multirow{5}{*}{2} & Spinal cord & - & - & - & 44.2 & 44.7 & 45.4 \\
\hline & Brainstem & - & - & - & 53.8 & 53.6 & 54.3 \\
\hline & Right parotid & 22.1 & 24.7 & 23.7 & - & - & - \\
\hline & Left parotid & 21.6 & 23.2 & 25.5 & - & - & - \\
\hline & Oral Cavity & 27.5 & 28.0 & 27.9 & - & - & - \\
\hline \multirow{5}{*}{3} & Spinal cord & - & - & - & 40.9 & 40.5 & 44.4 \\
\hline & Brainstem & - & - & - & 44.8 & 44.3 & 49.9 \\
\hline & Right parotid & 25.9 & 26.3 & 25.1 & - & - & - \\
\hline & Left parotid & 24.1 & 24.8 & 26.7 & - & - & - \\
\hline & Oral Cavity & 30.3 & 31.6 & 34.3 & - & - & - \\
\hline \multirow{5}{*}{4} & Spinal cord & - & - & - & 40.8 & 40.9 & 40.0 \\
\hline & Brainstem & - & - & - & 49.6 & 49.5 & 49.2 \\
\hline & Right parotid & 27.8 & 28.1 & 28.5 & - & - & - \\
\hline & Left parotid & 23.9 & 26.3 & 26.9 & - & - & - \\
\hline & Oral Cavity & 29.7 & 30.8 & 32.1 & 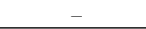 & - & - \\
\hline \multirow{5}{*}{5} & Spinal cord & - & - & - & 38.5 & 38.6 & 38.6 \\
\hline & Brainstem & - & - & - & 52.3 & 52.3 & 51.2 \\
\hline & Right parotid & 28.7 & 29.0 & 28.2 & - & - & - \\
\hline & Left parotid & 19.9 & 25.5 & 24.7 & - & - & - \\
\hline & Oral Cavity & 23.4 & 25.8 & 26.4 & - & - & - \\
\hline \multirow{5}{*}{6} & Spinal cord & - & - & - & 40.6 & 42.6 & 40.1 \\
\hline & Brainstem & - & - & - & 52.6 & 53.8 & 52.9 \\
\hline & Right parotid & 20.4 & 20.7 & 24.5 & - & - & - \\
\hline & Left parotid & 21.5 & 22.3 & 25.4 & - & - & - \\
\hline & Oral Cavity & 27.3 & 28.3 & 27.2 & - & - & - \\
\hline \multirow{5}{*}{7} & Spinal cord & - & - & - & 41.3 & 42.8 & 39.9 \\
\hline & Brainstem & - & - & - & 52.9 & 52.6 & 52.3 \\
\hline & Right parotid & 25.6 & 26.5 & 28.6 & - & - & - \\
\hline & Left parotid & 21.5 & 23.4 & 25.9 & - & - & - \\
\hline & Oral Cavity & 24.9 & 26.1 & 28.2 & - & - & - \\
\hline \multirow{5}{*}{8} & Spinal cord & - & - & - & 40.2 & 38.9 & 39.8 \\
\hline & Brainstem & - & - & - & 53.6 & 54.6 & 55.0 \\
\hline & Right parotid & 23.1 & 25.5 & 26.0 & - & - & - \\
\hline & Left parotid & 22.6 & 25.4 & 24.0 & - & - & - \\
\hline & Oral Cavity & 24.3 & 26.2 & 25.8 & - & - & - \\
\hline \multirow{5}{*}{9} & Spinal cord & - & - & - & 40.3 & 45.1 & 39.5 \\
\hline & Brainstem & - & - & - & 49.4 & 47.9 & 52.9 \\
\hline & Right parotid & 21.6 & 23.2 & 25.0 & - & - & - \\
\hline & Left parotid & 22.5 & 23.8 & 24.4 & - & - & - \\
\hline & Oral Cavity & 22.8 & 24.0 & 26.4 & - & - & - \\
\hline \multirow{5}{*}{10} & Spinal cord & - & - & - & 40.1 & 40.7 & 40.8 \\
\hline & Brainstem & - & - & - & 53.5 & 53.9 & 54.6 \\
\hline & Right parotid & 21.8 & 22.4 & 25.8 & - & - & - \\
\hline & Left parotid & 23.8 & 24.2 & 25.5 & - & - & - \\
\hline & Oral Cavity & 26.2 & 27.9 & 27.1 & - & - & - \\
\hline
\end{tabular}

start $B A O$ treatment plans achieve a mean dose irradiation reduction on the right parotid, left parotid and oral cavity of $2.1 \mathrm{~Gy}, 2.9$ Gy and 2.2 Gy, respectively. Furthermore, MultistartBAO treatment plans double the mean dose irradiation reduction numbers of Iterative $B A O$ treatment plans. Over-irradiation of salivary glands can lead to xerostomia, a common RT complication of nasopharyngeal cancer cases leading to swallow difficulties. Thus, the enhanced salivary glands sparing is of the utmost interest.

In clinical practice, results are typically judged by their dose-volume histogram (DVH). DVH are cumulative histograms that ideally would have $100 \%$ dose for the whole tumor volume dropping immediately to zero, while the curves for the remaining structures would be always zero meaning that no radiation was delivered to the healthy tissues. For illustration, DVH results for the fourth patient, a patient that obtained an average FMO value reduction, are displayed 
in Fig. 3. The DVH curves show enhanced tumor coverage and sparing by $\mathrm{Mul}$ tistartBAO treatment plans.

\section{Conclusions}

The $\mathrm{BAO}$ problem is a challenging highly non-convex optimization problem yet to be solved satisfactorily. Apart from iterative BAO, there is little or none commercial offer for beam direction selection. A parallel multistart PSM framework was presented and compared with iterative BAO using a set of clinical nasopharyngeal tumor cases. This multistart framework proved to be a competitive strategy to address the continuous $\mathrm{BAO}$ problem formulation. A global search scheme with a tailored sampling of the search space is combined with a procedure that locally improves the sampled ensembles. Despite the importance of the global strategy sketched, particularly for a search space with a peculiar shape due to symmetry properties, the choice of PSM, a derivative-free method, for locally improving the solutions is important to avoid local entrapment.

For the nasopharyngeal clinical cases retrospectively tested, the use of optimized directions obtained by the parallel multistart approach enhanced the quality of the treatment plans obtained. The high quality treatment plans obtained considering optimal beam ensembles were compared favorably with typical equispaced treatment plans. Furthermore, the presented multistart derivative-free framework for a continuous $\mathrm{BAO}$ formulation clearly outperforms an iterative approach for a combinatorial BAO formulation. Although iterative BAO reduces the number of comparisons required to achieve an improved solution compared to other combinatorial $\mathrm{BAO}$ approaches, it is a greedy strategy for the combinatorial $\mathrm{BAO}$ that truncates the search space at each iteration possibly disregarding the best ensembles with $n$-beam directions.

Several strategies for minimizing the number of function evaluations, and consequently decrease the computational time, were embedded in this multistart strategy including parallelization, searching in a reduced space and construction of hypercubes. In future work, further effort must be made to speed up even more this procedure maintaining the high quality results here detailed. One obvious strategy is to accelerate FMO computation as most of the BAO computational time is consumed for obtaining the optimal FMO values. Nevertheless, the computational burden of $\mathrm{BAO}$ will always decrease as future computer workstations will certainly become faster.

Acknowledgments. This work has been supported by project grant POCI-01-0145FEDER-028030 and by the Fundação para a Ciência e a Tecnologia (FCT) under project grant UID/MULTI/00308/2013.

\section{References}

1. Aleman, D.M., Kumar, A., Ahuja, R.K., Romeijn, H.E., Dempsey, J.F.: Neighborhood search approaches to beam orientation optimization in intensity modulated radiation therapy treatment planning. J. Global Optim. 42, 587-607 (2008) 




Fig. 3. Cumulative dose volume histogram comparing the results obtained by MultistartBAO, IterativeBAO and Equi for the fourth case.

2. Aleman, D.M., Romeijn, H.E., Dempsey, J.F.: A response surface approach to beam orientation optimization in intensity modulated radiation therapy treatment planning. INFORMS J. Comput. 21, 62-76 (2009)

3. Bangert, M., Ziegenhein, P., Oelfke, U.: Characterizing the combinatorial beam angle selection problem. Phys. Med. Biol. 57, 6707-6723 (2012)

4. Bangert, M., Ziegenhein, P., Oelfke, U.: Comparison of beam angle selection strategies for intracranial imrt. Med. Phys. 40, 011716 (2013)

5. Bertsimas, D., Cacchiani, V., Craft, D., Nohadani, O.: A hybrid approach to beam angle optimization in intensity-modulated radiation therapy. Comput. Oper. Res. 40, 2187-2197 (2013)

6. Breedveld, S., Storchi, P., Voet, P., Heijmen, B.: iCycle: integrated, multicriterial beam angle, and profile optimization for generation of coplanar and noncoplanar IMRT plans. Med. Phys. 39, 951-963 (2012)

7. Cheong, K., Suh, T., Romeijn, H., Li, J., Dempsey, J.: Fast Nonlinear Optimization with Simple Bounds for IMRT Planning. Med. Phys. 32, 1975-1975 (2005)

8. Craft, D.: Local beam angle optimization with linear programming and gradient search. Phys. Med. Biol. 52, 127-135 (2007)

9. Deasy, J.O., Lee, E.K., Bortfeld, T., Langer, M., Zakarian, K., Alaly, J., Zhang, Y., Liu, H., Mohan, R., Ahuja, R., Pollack, A., Purdy, J., Rardin, R.: A collaboratory for radiation theraphy planning optimization research. Ann. Oper. Res. 148, 55-63 (2006)

10. Dias, J., Rocha, H., Ferreira, B.C., Lopes, M.C.: Simulated annealing applied to IMRT beam angle optimization: A computational study. Phys. Med. 31, 747-756 (2015)

11. Dias, J., Rocha, H., Ventura, T., Ferreira, B.C., Lopes, M.C.: Automated fluence map optimization based on fuzzy inference systems. Med. Phys. 43, 1083-1095 (2016) 
12. Lim, G.J., Cao, W.: A two-phase method for selecting IMRT treatment beam angles: Branch-and-Prune and local neighborhood search. Eur. J. Oper. Res. 217, 609-618 (2012)

13. Monz, M., Kufer, K.H., Bortfeld, T.R., Thieke, C.: Pareto navigation Algorithmic foundation of interactive multi-criteria IMRT planning. Phys. Med. Biol. 53, 985998 (2008)

14. Rocha, H., Dias, J., Ferreira, B.C., Lopes, M.C.: Selection of intensity modulated radiation therapy treatment beam directions using radial basis functions within a pattern search methods framework. J. Glob. Optim. 57, 1065-1089 (2013)

15. Rocha, H., Dias, J., Ferreira, B.C., Lopes, M.C.: Beam angle optimization for intensity-modulated radiation therapy using a guided pattern search method. Phys. Med. Biol. 58, 2939-2953 (2013)

16. Rocha, H., Dias, J., Ferreira, B.C., Lopes, M.C.: Pattern search methods framework for beam angle optimization in radiotherapy design. Appl. Math. Comput. 219, 10853-10865 (2013)

17. Rocha, H., Dias, J., Ventura, T., Ferreira, B.C., Lopes, M.C.: A derivative-free multistart framework for an automated noncoplanar beam angle optimization in IMRT. Med. Phys. 43, 5514-5526 (2016)

18. Romeijn, H.E., Ahuja, R.K., Dempsey, J.F., Kumar, A., Li, J.: A novel linear programming approach to fluence map optimization for intensity modulated radiation therapy treatment planing. Phys. Med. Biol. 48, 3521-3542 (2003)

19. Wild, E., Bangert, M., Nill, S., Oelfke, U.: Noncoplanar VMAT for nasopharyngeal tumors: Plan quality versus treatment time. Med. Phys. 42, 2157-2168 (2015)

20. Yang, J., Zhang, P., Zhang, L., Shu, H., Li, B., Gui, Z.: Particle swarm optimizer for weighting factor selection in intensity-modulated radiation therapy optimization algorithms. Phys. Med. 33, 136-145 (2017) 